\title{
Fractional order inductive phenomena based on the skin effect
}

\author{
J.A. Tenreiro Machado • \\ Alexandra M.S.F. Galhano
}

\begin{abstract}
The Maxwell equations play a fundamental role in the electromagnetic theory and lead to models useful in physics and engineering. This formalism involves integer-order differential calculus, but the electromagnetic diffusion points towards the adoption of a fractional calculus approach. This study addresses the skin effect and develops a new method for implementing fractional-order inductive elements. Two genetic algorithms are adopted, one for the system numerical evaluation and another for the parameter identification, both with good results.
\end{abstract}

Keywords Skin effect - Electromagnetism . Inductance $\cdot$ Fractional calculus $\cdot$ Genetic algorithms

\section{Introduction}

The tendency of a high-frequency electric current to distribute itself in a conductor so that the current density near the surface is greater than that at its core is called the skin effect (SE). The SE can be reduced by using stranded instead of solid wire, increasing the effective surface area of the wire for a given wire gauge.
This phenomenon shows characteristics well modelled by the Fractional Calculus (FC) tools [1, 15, 17, $18,20]$ revealing a dynamics of half order. Moreover, the model development based on Maxwell's equations suggests the possibility of implementing devices with inductive characteristics of variable fractional order. This characteristic is of utmost importance since researchers have been devoting attention mainly to the electrical elements with fractional-order capacitances $[5,6,11,22]$, while the inductive case has been addressed only as an effect occurring in some electrical machines [2, 4, 14, 21].

Having these ideas in mind this paper is organized as follows. Section 2 summarizes the classical mathematical description of the SE based on the Maxwell equations. Section 3 re-evaluates the results demonstrating its fractional dynamics of half order. After clarifying the fundamental concepts Sect. 4 addresses the case of implementing inductive electrical elements of variable fractional order. For that purpose two genetic algorithms are adopted. A first genetic algorithm is adopted for yielding the initial conditions required in the calculation of the model differential equations. The second genetic algorithm is used for identifying the parameters of a FC approximate model. Finally, Sect. 5 draws the main conclusions.

\section{The skin effect}

In the differential form the Maxwell equations are [19] 
$\nabla \times \mathbf{E}=-\frac{\partial \mathbf{B}}{\partial t}$

$\nabla \times \mathbf{H}=\boldsymbol{\delta}+\frac{\partial \mathbf{D}}{\partial t}$

$\nabla \cdot \mathbf{D}=\rho$

$\nabla \cdot \mathbf{B}=0$

where $\mathbf{E}, \mathbf{D}, \mathbf{H}, \mathbf{B}, \delta$ represent the vectors of electric field intensity, electric flux density (or electric displacement), magnetic field intensity, magnetic flux density and the current density, respectively, $\rho$ and $t$ are the charge density and time, and $\nabla$ is the nabla operator.

For a homogeneous, linear and isotropic media, we have

$\mathbf{D}=\varepsilon \mathbf{E}$

$\mathbf{B}=\mu \mathbf{H}$

$\boldsymbol{\delta}=\gamma \mathbf{E}$

where $\varepsilon, \mu$ and $\gamma$ are the electrical permittivity, the magnetic permeability and the conductivity, respectively.

In order to study the SE we consider a cylindrical conductor with radius $r_{0}$ conducting a current $I$ along its longitudinal axis. In a conductor, even for high frequencies, the term $\frac{\partial \mathbf{D}}{\partial t}$ is negligible in comparison with the conduction term $\delta$, that is, the displacement current is much lower than the conduction current, and (2) simplifies to $\nabla \times \mathbf{H}=\boldsymbol{\delta}$. Therefore, for a radial distance $r<r_{0}$ the application of Maxwell's equations with the simplification of (2) leads to the expression $[3,12]$ :

$\nabla \times(\nabla \times \delta)=-\gamma \mu \frac{\partial \boldsymbol{\delta}}{\partial t}$

Knowing that $\nabla \times(\nabla \times \boldsymbol{\delta})=\nabla(\nabla \cdot \boldsymbol{\delta})-\nabla^{2} \boldsymbol{\delta}$, where $\nabla^{2}$ is the Laplacian, and that $\nabla \cdot \boldsymbol{\delta}=0$ we have

$\nabla^{2} \boldsymbol{\delta}=\gamma \mu \frac{\partial \boldsymbol{\delta}}{\partial t}$

For cylindrical coordinates and for $\delta$ pointing in the direction of the $z$ axis the Laplacian can be expressed as $\nabla^{2} \delta=\frac{1}{r} \frac{\partial}{\partial r}\left(r \frac{\partial \delta}{\partial t}\right)$. Therefore, rewriting (9) in terms of the electric field intensity $E$ it becomes

$\frac{\partial^{2} E}{\partial r^{2}}+\frac{1}{r} \frac{\partial E}{\partial r}=\gamma \mu \frac{\partial E}{\partial t}$
For a sinusoidal field $E=\sqrt{2} \tilde{E} \sin (\omega t)$ we adopt the complex notation $E=\sqrt{2} \tilde{E} e^{j \omega t}$, where $j=\sqrt{-1}$, yielding

$\frac{d^{2} \tilde{E}}{d r^{2}}+\frac{1}{r} \frac{d \tilde{E}}{d r}+q^{2} \tilde{E}=0$

with $q^{2}=-j \omega \gamma \mu$.

Equation (11) is a particular case of the Bessel equation that has a solution:

$\widetilde{E}=\frac{q}{2 \pi r_{0} \gamma} \frac{J_{0}\left(q r_{0}\right)}{J_{1}\left(q r_{0}\right)} I, \quad 0 \leq r \leq r_{0}$

where $J_{0}$ and $J_{1}$ are complex valued Bessel functions of the first kind of orders 0 and 1, respectively.

Equation (12) establishes the SE phenomenon that consists on having a non-uniform current density, namely a low density near the conductor axis and an high density on surface, the higher the frequency $\omega$. Therefore, for a conductor of length $l_{0}$ the total voltage drop is $\tilde{Z} \tilde{I}=\tilde{E} l_{0}$ and the equivalent electrical complex impedance $\widetilde{Z}$ is given by

$\widetilde{Z}=\frac{q l_{0}}{2 \pi r_{0} \gamma} \frac{J_{0}\left(q r_{0}\right)}{J_{1}\left(q r_{0}\right)}$

For small values of $x$ the Taylor series [16] leads to

$J_{0}(x)=1-\frac{x^{2}}{2^{2}}+\cdots$

$J_{1}(x)=\frac{x}{2}-\frac{x^{3}}{2^{2} 4}+\cdots$

For large values of $x$ the asymptotic expansion yields

$$
\begin{gathered}
J_{n}(x)=\sqrt{\frac{2}{\pi x}} \cos \left(x-n \frac{\pi}{2}-\frac{\pi}{4}\right), \\
n=0,1, \ldots
\end{gathered}
$$

Knowing (14), (15) and (16), the low- and highfrequency approximations of $\widetilde{Z}$ result:

$$
\begin{aligned}
& \omega \rightarrow 0 \quad \Rightarrow \quad \tilde{Z} \rightarrow \frac{l_{0}}{\pi r_{0}^{2} \gamma} \\
& \omega \rightarrow \infty \quad \Rightarrow \quad \tilde{Z} \rightarrow \frac{l_{0}}{2 \pi r_{0}} \sqrt{\frac{\omega \mu}{2 \gamma}}(1+j)
\end{aligned}
$$


Fig. 1 Variation of $R$ and $L$ for the integer-order model $\tilde{Z}=R+j \omega L$ with $\gamma=10^{7} \Omega^{-1} \mathrm{~m}, l_{0}=1 \mathrm{~m}$, $r_{0}=3.02 \times 10^{-3} \mathrm{~m}$, $\mu_{0}=1.257 \times 10^{-6} \mathrm{Hm}^{-1}$ and $\mu_{r}=10^{3}$

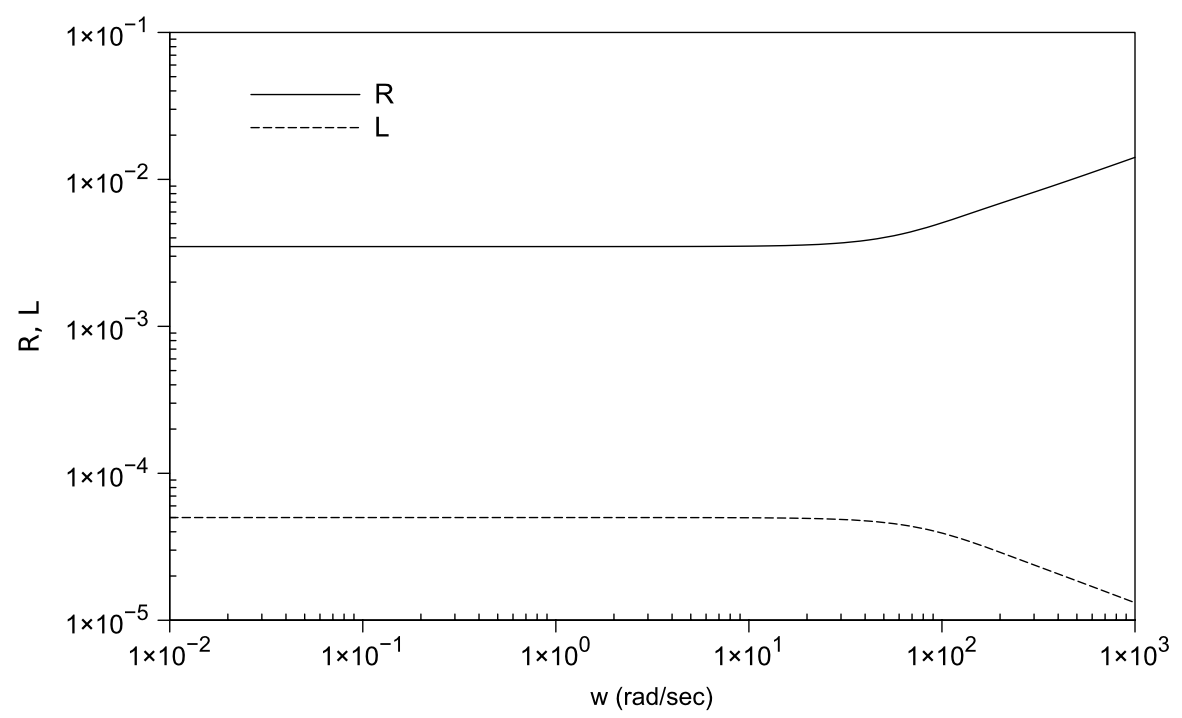

\section{The fractional calculus perspective}

The standard approach in electrical engineering in order to avoid handling the transcendental equation (13) is to assign a resistance $R$ and an inductance $L$ given by $\tilde{Z}=R+j \omega L$. Nevertheless, this method is inadequate because the model parameters $\{L, R\}$ must vary with the frequency (see Fig. 1).

Expression (18) reveals the half-order nature of the dynamic phenomenon, at high frequencies (i.e., $\tilde{Z} \sim \omega^{\frac{1}{2}}$ ) which is not captured by the classical integerorder approach. The FC eliminates those problems $[9,13]$. A simple method is to join the two asymptotic expressions (17)-(18) through the so-called explicit and implicit fractional-order approximations:

$$
\begin{aligned}
& \tilde{Z}_{\text {app }}=Z_{0}\left[1+\left(\frac{j \omega}{a}\right)^{\alpha}\right] \\
& \tilde{Z}_{\text {app }}=Z_{0}\left(1+\frac{j \omega}{a}\right)^{\alpha}
\end{aligned}
$$

where $Z_{0}=\frac{l_{0}}{\pi r_{0}^{2} \gamma}, a=\frac{4}{r_{0}^{2} \gamma \mu}$ and $\alpha=\frac{1}{2}$. It must be noted that, while other approximations are possible, expressions (19)-(20) have simple analytical structures yielding $\tilde{Z}_{\text {app }}=\frac{l_{0}}{\pi r_{0}^{2} \gamma}$ and $\tilde{Z}_{\text {app }}=\frac{l_{0}}{2 \pi r_{0}} \sqrt{\frac{\omega \mu}{2 \gamma}}(1+$ $j$ ) for $\omega \rightarrow 0$ and $\omega \rightarrow \infty$, respectively.

For example, Fig. 2 compares the Bode diagrams of amplitude and phase of $E\left(k_{0}\right)$ based on expressions (13), (19) and (20) in the case of a conductor with $\gamma=10^{7} \Omega^{-1} \mathrm{~m}, l_{0}=1 \mathrm{~m}, r_{0}=3.02 \times 10^{-3} \mathrm{~m}$, $\mu_{0}=1.257 \times 10^{-6} \mathrm{Hm}^{-1}$ and $\mu_{r}=10^{3}$. For model (13) the numerical values were obtained through a symbolic mathematical package, while for (19)-(20) it is straightforward to attain the results. We verify that the explicit (19) is inferior to the implicit (20) fractional-order approximation, and that this one leads to a very good curve fitting.

\section{Fractional-order inductive elements}

In the previous section we observed that the SE leads to a fractional model of the electrical field of order $\alpha=\frac{1}{2}$. A possible question is if, and how, other values of $\alpha$ can be designed, either by varying the geometry of the conductor, or by modifying its electromagnetic properties.

Equation (11) has now to be integrated numerically since it does not follow any more the Bessel equation. Adopting the Euler forward approximation for the first and second order derivatives and making explicit the real and imaginary components of the sinusoidal electric field, $E=E_{r}+j E_{i}$, we get the approximation

$$
\begin{aligned}
& E_{r}(k+2)+\left(-2+\frac{\Delta r}{r}\right) E_{r}(k+1) \\
& +\left(1-\frac{\Delta r}{r}\right) E_{r}(k)+\omega \mu \Delta r^{2} \gamma(r) E_{i}(k)=0
\end{aligned}
$$


Fig. 2 Amplitude and phase Bode diagrams of $E\left(k_{0}\right)$ for the theoretical and the approximate expressions with $\gamma=10^{7} \Omega^{-1} \mathrm{~m}, l_{0}=1 \mathrm{~m}$, $r_{0}=3.02 \times 10^{-3} \mathrm{~m}$, $\mu_{0}=1.257 \times 10^{-6} \mathrm{Hm}^{-1}$ and $\mu_{r}=10^{3}$
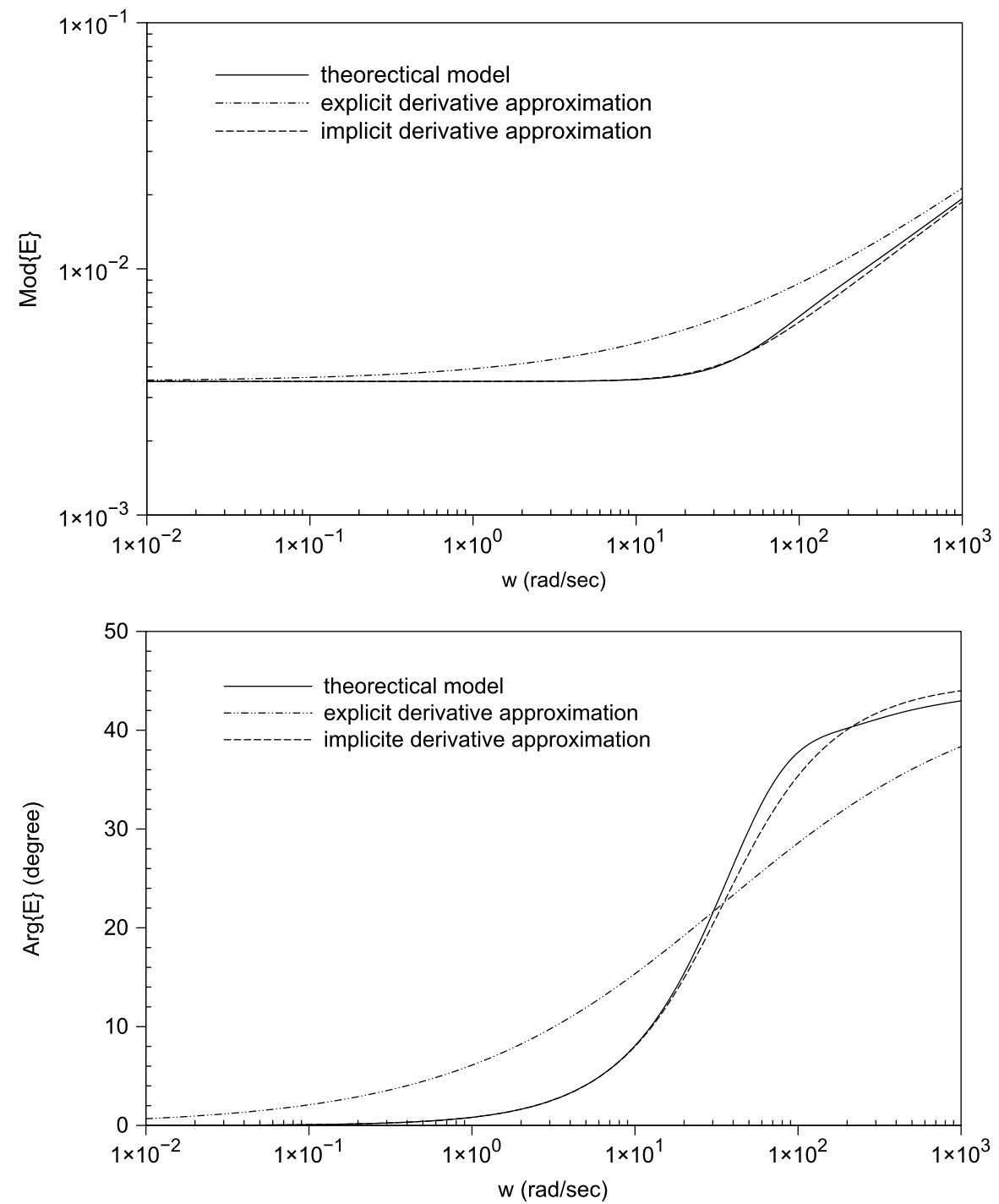

$$
\begin{aligned}
& E_{i}(k+2)+\left(-2+\frac{\Delta r}{r}\right) E_{i}(k+1) \\
& \quad+\left(1-\frac{\Delta r}{r}\right) E_{i}(k)-\omega \mu \Delta r^{2} \gamma(r) E_{r}(k)=0
\end{aligned}
$$

where $k$ and $k+1$ represent two consecutive sampling points in space and $\Delta r$ is the integration step along the conductor radius.

The numerical initialization must be obtained from the boundary conditions

$$
\begin{aligned}
& H=\frac{1}{j \omega \mu} \frac{d E}{d r} \\
& H_{0}=H\left(r=r_{0}\right)=\frac{I}{2 \pi r_{0}}
\end{aligned}
$$

The calculation of (21)-(22) requires initial conditions compatible with (23)-(24). Therefore, for estimating the (unknown) initial conditions it was implemented a Genetic Algorithm (GA) [7, 8, 10], whose population are the values $\left\{E_{r}(1), E_{r}(0), E_{i}(1), E_{i}(0)\right\}$, with fitness function:

$$
\begin{aligned}
J_{\text {init }}= & {\left[\frac{1}{\omega \mu} \frac{E_{i}(k+1]-E_{i}(k)}{\Delta r}\right]^{2} } \\
& +\left[E_{r}(k+1)-E_{r}(k)\right]^{2} \\
H_{0}= & \frac{1}{\omega \mu} \frac{E_{i}\left(k_{0}\right)-E_{i}\left(k_{0}-1\right)}{\Delta r}
\end{aligned}
$$

where $k_{0} \Delta r=r_{0}$. It was adopted a GA population of $n_{\mathrm{GA}}=2000$ elements, crossover and muta- 
Fig. 3 Amplitude and phase Bode diagrams of $E\left(k_{0}\right)$ for a annular conductor with inner and outer radius $r_{1}$ and $r_{0}$, such that

$r_{1}=\{0,0.3,0.5,0.7\} \times r_{0}$, with $\gamma=10^{7} \Omega^{-1} \mathrm{~m}$,

$l_{0}=1 \mathrm{~m}$,

$r_{0}=3.02 \times 10^{-3} \mathrm{~m}$,

$\mu_{0}=1.257 \times 10^{-6} \mathrm{Hm}^{-1}$ and $\mu_{r}=10^{3}$
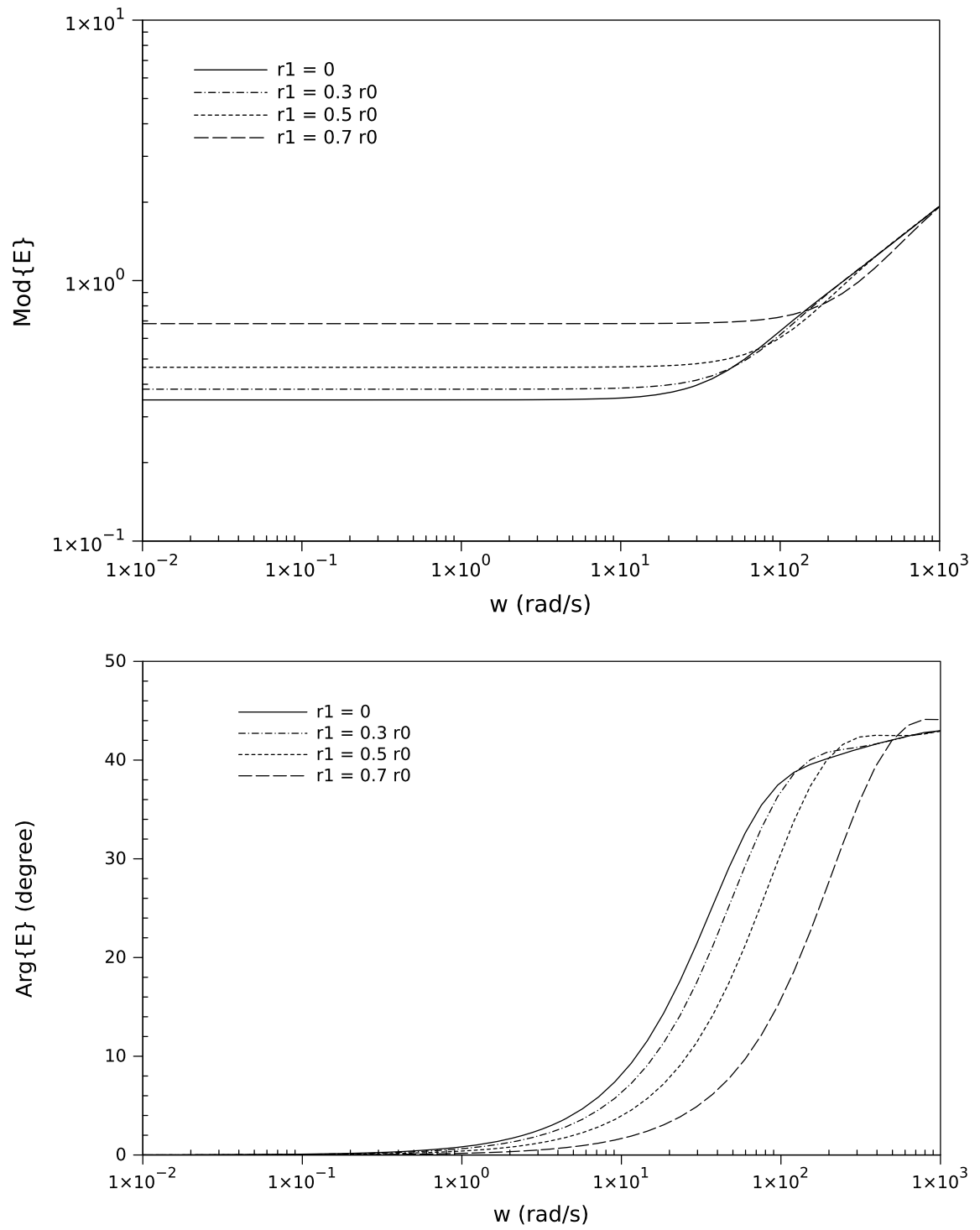

tion probabilities of $p_{c}=0.5$ and $p_{m}=0.1$, respectively, and elitism. Furthermore, the GA was executed during $I_{\mathrm{GA}}=2000$ iterations for a total of $n_{\Omega}$ testing frequencies, logarithmically spaced in the interval $\omega_{\min }<\omega<\omega_{\max }$, of the sinusoidal electric field $E=$ $\sqrt{2} \tilde{E} \sin (\omega t)$. In the numerical experiments was considered a set of frequencies $\Omega$ such that $\omega_{\min }=10^{-2}$, $\omega_{\max }=10^{3}$ and $n_{\Omega}=30$.

Expressions (25)-(26) pose a large computational load, for the initialization GA. In order to reduce the burden the integration step $\Delta r$ was adjusted, from gross to fine, during three phases in the GA evolution, namely
$\Delta r(i)= \begin{cases}\frac{r_{0}}{66} & i \leq \frac{1}{3} n_{\mathrm{GA}} \\ \frac{r_{0}}{133} & \frac{1}{3} n_{\mathrm{GA}}<i \leq \frac{2}{3} n_{\mathrm{GA}} \\ \frac{r_{0}}{200} & \frac{2}{3} n_{\mathrm{GA}}<i \leq n_{\mathrm{GA}}\end{cases}$

where the index $i$ represents the GA generation.

This algorithm guarantees the convergence of the numerical integration of (21)-(22) and leads to a reduction of $50 \%$ in the computational burden of the fitness evaluation. In fact, several experiments, comparing the results of the variable and the fixed step sizes, demonstrated the feasibility of the proposed calculation scheme. 
Fig. 4 Amplitude and phase Bode diagrams of $E\left(k_{0}\right)$ for $\gamma=10^{7} \Omega^{-1} \mathrm{~m}$, $r_{0}=3.02 \times 10^{-3} \mathrm{~m}$, $\mu_{0}=1.257 \times 10^{-6} \mathrm{Hm}^{-1}$, $\mu_{r}=10^{3}$ and $\beta=\left\{-1,-\frac{1}{2}, 0, \frac{1}{2}, 1\right\}$
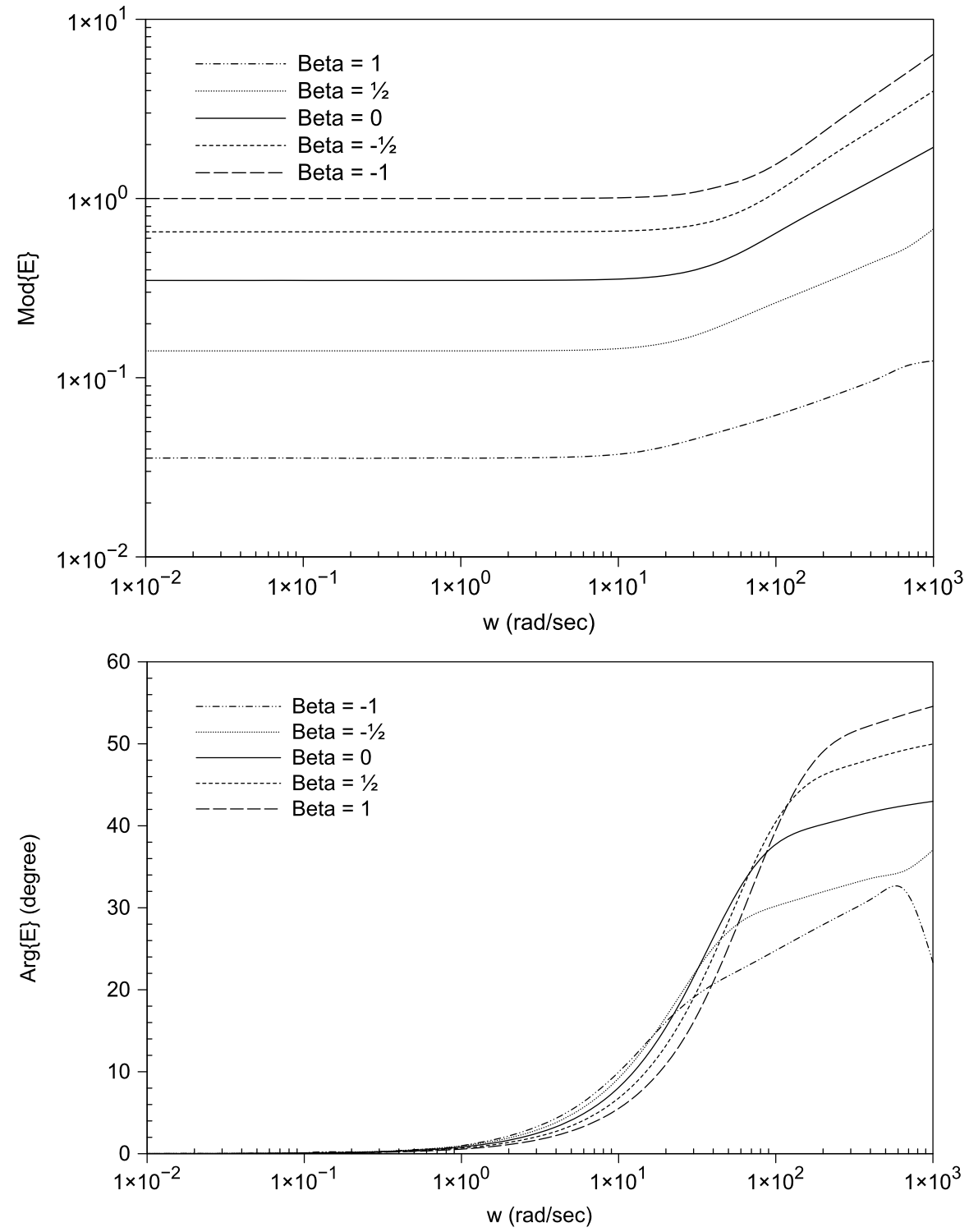

The first approach for modifying the properties of the SE consists of adopting a conductor with a different geometry. One simple possibility is, for example, to have a annular conductor with inner and outer radius $r_{1}$ and $r_{0}$, respectively. Figure 3 shows the amplitude and phase Bode diagrams of $E\left(k_{0}\right)$ for $r_{1}=$ $\{0,0.3,0.5,0.7\} \times r_{0}$. We verify that by eliminating the flow of current in the inner part of the conductor we can shift the frequency response.

The second approach for a different SE consists of varying the electrical conductivity with the conductor radial distance, that is, to have $\gamma=\gamma(r), 0 \leq r \leq r_{0}$. For the electrical conductivity the following expres- sion was considered:

$\gamma(r)=\gamma_{0}\left(1-\frac{r}{r_{0}}\right)^{\beta}, \quad-\infty<\beta<+\infty$

Obviously, $\beta=0$ yields the case of constant electrical conductivity which was analyzed analytically in Sects. 2 and 3.

During the experiments the numerical values $\gamma=$ $10^{7} \Omega^{-1} \mathrm{~m}, r_{0}=3.02 \times 10^{-3} \mathrm{~m}, \mu_{0}=1.257 \times$ $10^{-6} \mathrm{Hm}^{-1}, \mu_{r}=10^{3}, \omega_{\min }=10^{-2} \mathrm{~s}^{-1}$ and $\omega_{\max }=$ $10^{3} \mathrm{~s}^{-1}$ were adopted. 
Fig. 5 Variation of the parameters $\left\{E_{0}, a, \alpha\right\}$ versus $\beta$
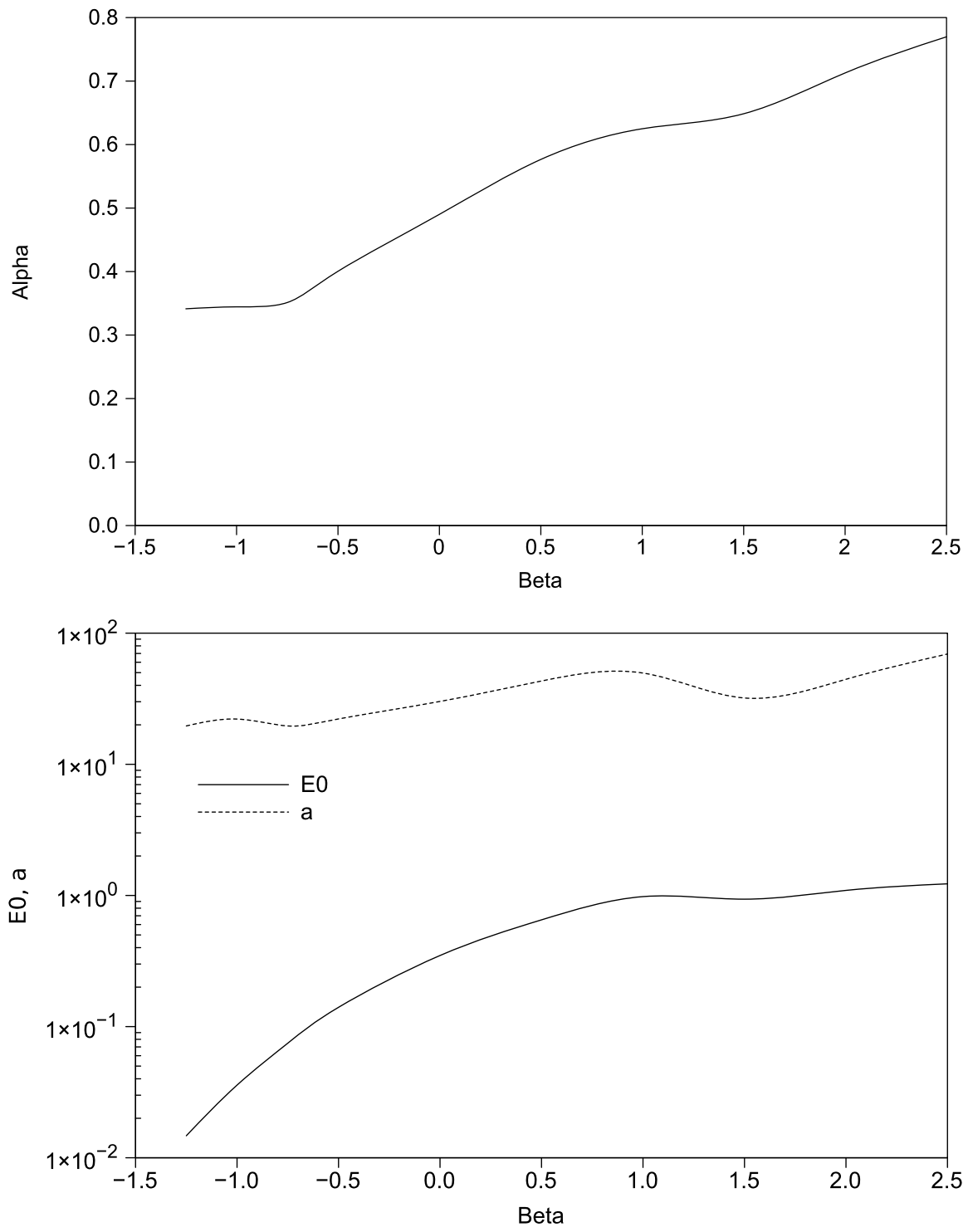

Figure 4 depicts the Bode diagrams of amplitude and phase of $E\left(k_{0}\right)$ for $\beta=\left\{-1,-\frac{1}{2}, 0, \frac{1}{2}, 1\right\}$.

It was observed that when moving far away from the central case of $\beta=0$ the results became more and more 'unstable', that is, with considerable variations in the plots. Therefore, in the study were considered only those cases that depicted a sound numerical response.

The Bode plots reveal that at low frequencies we get the usual resistive behaviour, but at high frequencies we have inductive effects of different fractional order. Therefore, it was decided to approximate the numerical results by expressions of the type

$$
\begin{aligned}
E_{\text {app }} & =E_{0}\left(1+\frac{j \omega}{a}\right)^{\alpha}, \\
E_{0} & >0, a>0, \alpha>0
\end{aligned}
$$

For the estimation of the three parameters $\left\{E_{0}, a, \alpha\right\}$ an identification GA with fitness function:

$$
\begin{aligned}
J_{\text {ident }}= & \sum\left[\operatorname{Re}\left(E_{\text {app }}\right)-E_{r}\left(k_{0}\right)\right]^{2} \\
& +\left[\operatorname{Im}\left(E_{\text {app }}\right)-E_{i}\left(k_{0}\right)\right]^{2}
\end{aligned}
$$

was implemented, where $\Omega$ represents the set of $n_{\Omega}$ sampling frequencies such that $\omega_{\min }<\omega<\omega_{\max }$. The parameters of the identification GA are: population of 
$n_{\mathrm{GA}}=5000$ elements, crossover and mutation probabilities of $p_{c}=0.5$ and $p_{m}=0.1$, respectively, and elitism. The GA was executed during $I_{\mathrm{GA}}=1000$ iterations, for $\omega_{\min }=10^{-2}, \omega_{\max }=10^{3}$ and $n_{\Omega}=30$.

Figure 5 depicts the parameters $\left\{E_{0}, a, \alpha\right\}$ of expression (29) versus $\beta$. We verify clearly that by varying the electrical conductivity in (28) we can design different fractional orders $\alpha$.

Several aspects should be highlighted. Firstly, we note that while ongoing research in FC focuses often in the development of fractional-order capacitors $[5,6,11,22]$, this study addresses the dual element, a fractional-order inductance. Secondly, we must note that the effect of the modification of the conductor cross section and its conductivity can be further tested, either separately or simultaneously. For example, the adoption of conductors having surfaces with different type of roughness remains to be explored.

\section{Conclusions}

The classical electromagnetism and the Maxwell equations lead to models requiring a FC perspective to be fully interpreted. Nevertheless, in the standard studies of electromagnetic diffusion we get only halforder effects. This paper addressed the implementation of inductive elements of any fractional order based on the SE. It was demonstrated that by designing the variation of the conductor electrical conductivity we can get different fractional orders of the SE. The adoption of GAs simplified considerably the calculations, both for the initialization of the numerical integration algorithm and the identification of the system parameters. The results point towards the technological implementation of devices with the fractional characteristics.

\section{References}

1. Kilbas, A.A., Srivastava, H.M., Trujillo, J.J.: Theory and Applications of Fractional Differential Equations. NorthHolland Mathematics Studies, vol. 204. Elsevier, Amsterdam (2006)

2. Benchellal, A., Poinot, T., Bachir, S., Trigeassou, J.C.: Identification of a non-integer model of induction machines. In: Proc. 1st IFAC Workshop on Fractional Differentiation and Its Applications, Bordeaux, France, pp. 400407 (2004)

3. Bessonov, L.: Applied Electricity for Engineers. MIR Publishers, Moscow (1968)
4. Bidan, P.: Commande diffusive d'une machine electrique: une introduction. In: ESAIM Proceedings, France, vol. 5, pp. 55-68 (1998)

5. Bohannan, G.W.: Analog realization of a fractional control element-revisited. In: Proc. of the 41st IEEE Int. Conf. on Decision and Control, Tutorial Workshop 2: Fractional Calculus Applications in Automatic Control and Robotics, Las Vegas, NV (2002)

6. Carlson, G.E., Halijak, C.A.: Approximation of fractional capacitors $(1 / s)^{(1 / n)}$ by a regular newton process. IEEE Trans. Circuit Theory CT-10(2), 210-213 (1964)

7. Goldenberg, D.E.: Genetic Algorithms in Search Optimization, and Machine Learning. Addison-Wesley, Reading (1989)

8. Holland, J.H.: Adaptation in Natural and Artificial Systems. University of Michigan Press, Ann Arbor (1975)

9. Tenreiro Machado, J.A., Galhano, A., Boaventura, G.J., Jesus, I.S.: Fractional calculus analysis of the electrical skin phenomena. In: Sabatier, J., Agrawal, O.P., Tenreiro Machado, J. (eds.) Advances in Fractional Calculus: Theoretical Developments and Applications in Physics and Engineering, pp. 323-332. Springer, Dordrecht (2007)

10. Tenreiro Machado, J.A., Alexandra, A.M.O., Galhano, M., Tar, J.K.: Optimal approximation of fractional derivatives through discrete-time fractions using genetic algorithms. Commun. Nonlinear Sci. Numer. Simul. 15(3), 482-490 (2010)

11. Jesus, I.S., Machado Tenreiro, J.A.: Development of fractional order capacitors based on electrolyte processes. Nonlinear Dyn. 56(1-2), 45-55 (2009)

12. Küpfmüller, K.: Einführung in die Theoretische Elektrotechnik. Springer, Berlin (1939)

13. Machado, J.A.T., Jesus, I.S.: A suggestion from the past? Fract. Calc. Appl. Anal. 7(4), 403-407 (2004)

14. Malpica, W.A., Tenreiro Machado, J., Silva, J.F., de Barros, M.T.C.: Fractional order calculus on the estimation of short-circuit impedance of power transformers. In: Proc. 1st IFAC Workshop on Fractional Differentiation and Its Applications, Bordeaux, France, pp. 408-415 (2004)

15. Miller, K.S., Ross, B.: An Introduction to the Fractional Calculus and Fractional Differential Equations. Wiley, New York (1993)

16. Milton, A., Stegun, I.A.: Handbook of Mathematical Functions with Formulas, Graphs, and Mathematical Tables. Dover, New York (1965)

17. Oldham, K.B., Spanier, J.: The Fractional Calculus: Theory and Application of Differentiation and Integration to Arbitrary Order. Academic Press, New York (1974)

18. Podlubny, I.: Fractional Differential Equations. An Introduction to Fractional Derivatives, Fractional Differential Equations, to Methods of Their Solution. Mathematics in Science and Engineering, vol. 198. Academic Press, San Diego (1998)

19. Richard, P., Feynman, R.B.L., Sands, M.: The Feynman Lectures on Physics: Mainly Electromagnetism and Matter. Addison-Wesley, Reading (1964)

20. Samko, S.G., Kilbas, A.A., Marichev, O.I.: Fractional Integrals and Derivatives: Theory and Applications. Gordon and Breach, Amsterdam (1993) 
21. Sylvain, C., Faucher, J.: Fractional order: Frequential parametric identification of the skin effect in the rotor bar of squirrel cage induction machine. In: Proc. of the ASME 2003 Design Engineering Technical Conf. and Computers and Information in Engineering Conf., Chicago, IL, DETC 2003/VIB-48393 (2003)

22. Westerlund, S., Ekstam, L.: Capacitor theory. IEEE Trans. Dielectr. Electr. Insul. 1(5), 826-839 (1994) 